\title{
Nordiques
}

$37 \mid 2019$

Mythes et réalités de l'Arctique

\section{L'histoire hermaphrodite : sur les Groenlands de Kim Leine}

\section{Christian Bank Pedersen}

\section{(2) OpenEdition \\ 1 Journals}

Édition électronique

URL : http://journals.openedition.org/nordiques/439

DOI : 10.4000/nordiques.439

ISSN : $2777-8479$

Éditeur :

Association Norden, Bibliothèque de Caen la mer

Édition imprimée

Date de publication : 1 mai 2019

Pagination : $57-75$

ISBN : 979-1-0959140-3-7

ISSN : $1761-7677$

Référence électronique

Christian Bank Pedersen, "L'histoire hermaphrodite : sur les Groenlands de Kim Leine », Nordiques [En ligne], 37 | 2019, mis en ligne le 31 octobre 2020, consulté le 13 mars 2021. URL : http://

journals.openedition.org/nordiques/439; DOI : https://doi.org/10.4000/nordiques.439 


\section{L'histoire hermaphrodite : sur les Groenlands de Kim Leine}

Christian Bank Pedersen*

\section{RÉSUMÉ}

Dans l'ouvre de Kim Leine, l'écriture historico-sociale - c'est-à-dire la réflexion romanesque sur une réalité historique ou contemporaine - sarticule dans une relation étroite avec la quête autobiographique de l'identité à travers la littérature. Tout au long du présent article, j'étudierai en ce sens les différentes perspectives que propose Leine sur l'histoire coloniale qui lie le Groenland et le royaume de Danemark, en mettant l'accent sur le roman Les prophètes du fjord de l'Éternité. Dans ce contexte, la figure de lhermaphrodite sera de première importance, car elle englobe pour l'auteur tous les niveaux décisifs de la réflexion: chez Leine, cet équivalent mythologique de la contradictio in terminis logique incarne symboliquement la complexité de la question de l'identité saisie en tant que dualité, et ce, tant au niveau individuel qu'au niveau historique et culturel collectif, entre liberté et dépendance.

\section{ABSTRACT}

In the work of Kim Leine, the novelistic approach to historical or contemporary reality is articulated in close relation to the autobiographical quest for identity through literature. In my article, I will explore Leine's different perspectives on the colonial history linking Greenland to the Kingdom of Denmark. Throughout, I will be focusing on the novel The Prophets of Eternal Fjord. In this context, the figure of the hermaphrodite is of major importance. Indeed, in the vision of the author it concentrates all the decisive levels of reflection: to Leine, this mythological equivalent of the logical contradictio in terminis symbolically embodies the complexity of the question of identity as duality, oscillating between freedom and dependence, both at the individual level and at the collective level of history and culture.

* Maître de conférences, Département d'études nordiques, université de Caen Normandie. Membre de l'EA 4254 ERLIS. 
Lauteur dano-norvégien Kim Leine (1961-) compte aujourd'hui parmi les romanciers les plus importants de la Scandinavie, couvrant un registre thématique, historique et artistique très vaste, des malheurs les plus dévastateurs de la vie familiale à la question de la capacité de l'écriture littéraire à représenter l'histoire : après ses débuts en 2007 avec Kalak, un " roman de mémoires " sur son éducation religieuse et les abus sexuels dont il fut victime adolescent, ainsi que sur l'alcoolisme et la toxicomanie dans lesquels il est tombé lors d'un long séjour au Groenland, Leine publie Jour de Valdemar (2008), récit d'un meurtre commis à Copenhague, le 15 juin 1938, et ce, apparemment par le grand-père paternel de l'auteur. Dans Tunu (2009), roman collectif sur la société groenlandaise actuelle, il considère la situation de ce "territoire rattaché " au royaume de Danemark qui cherche encore aujourd'hui les moyens de définir son indépendance, après presque trois siècles de colonisation, assortis depuis quelques décennies d'une certaine autonomie. Ensuite, il change de voie en se tournant vers la forme du roman historique, d'abord avec Les prophètes du ford de l'Eternité (2012), qui relate le parcours fictif d'un pasteur errant, à la fin du XVIII siècle, entre le Danemark et le Groenland, puis à travers L'abîme (2015), sur l'histoire $\mathrm{du}$ Nord de la première moitié du XX $\mathrm{X}^{\mathrm{e}}$ siècle, histoire vue par le biais du destin imaginaire de deux frères jumeaux danois, de leur participation volontaire du côté des Blancs lors de la guerre d'indépendance de Finlande, en 1918, jusqu’à leur entrée dans la résistance danoise au cours de la Seconde Guerre mondiale. En 2016, Leine se projette dans l'écriture dystopique en sortant Les insomniaques, récit futuriste d'une journée dans un hôpital groenlandais en 2025, sur fond de guerre en Europe. Tout récemment, au printemps 2018, l'auteur a fait paraître le second tome de sa trilogie historique - encore inachevée - sur la colonisation danoise du Groenland : faisant suite aux Prophètes du ford de l'Éternité, Homme rouge/Homme noir se penche sur les premières décennies de cette colonisation en scrutant les relations conflictuelles et les filiations secrètes de deux hommes que visiblement tout oppose, le pasteur colonisateur dano-norvégien Hans Egede, l'« homme noir ", et le chaman groenlandais Aappaluttoq, l'« homme rouge ».

\section{INDIVIDU ET HISTOIRE, VICTIME ET BOURREAU}

Deux approches étroitement liées déterminent l'œuvre de Kim Leine : l'écriture historico-sociale - c'est-à-dire la réflexion romanesque sur une réalité historique ou contemporaine - est indissociable de la quête autobiographique de l'identité à travers la littérature et donc de la question de " devenir celui qu'on est ", pour reprendre la formule de Nietzsche ${ }^{1}$. Citons un passage du premier roman de l'auteur, Kalak. Dans celui-ci, le narrateur fait face aux méandres grammaticaux

1 Dans le sous-titre de l'écrit autobiographique Ecce homo. Wie man wird, was man ist, paru à titre posthume en 1908 . 
et syntaxiques de la langue groenlandaise. Par là, il expose implicitement les principaux thèmes de cette œuvre, des romans à venir et de l'écriture de Leine, à savoir les liens apparemment contraires entre le passé et le présent - "passé " et " présent » individuels et historiques -, et entre le bourreau et sa victime, le maître et son esclave.

J'essaie de calculer combien de formes fléchies un verbe peut avoir et j'arrive à 640. Par ailleurs, les verbes se comportent fréquemment comme des substantifs, les substantifs comme des adjectifs et les adjectifs comme des verbes, ce qui complique évidemment les choses. Une phrase avec sujet, verbe et complément d'objet indirect est le plus souvent constituée d'un seul mot, qui est pour sa part composé de chaînes de cinq à dix syllabes. Le prétérit ordinaire n'existe pas. On dit : «Je le rencontre hier ». En revanche, on a le plus-que-parfait, le futur, le futur hypothétique, le plus-que-parfait transitif hypothétique négatif, etc. «Si je n’avais pas eu à le rencontrer ». Naappissanngikkaluarukku. ${ }^{2}$

Pour un locuteur de langue maternelle indo-européenne, le groenlandais - kalaallisut, "la langue du peuple ", qui désigne le groenlandais officiel, parlé sur la côte ouest de l'île -, n'est que difficilement maîtrisable. Et tout est effectivement question de maîtrise, du niveau grammatical jusqu'au niveau politique. Pourtant, il essaie, le maître danois qui porte la voix, ce narrateur qui occupe pendant quinze ans - par intermittence - un poste d'infirmier au sein de différentes structures de santé groenlandaises et qui est donc souvent amené à soigner les conséquences médicales des fléaux sociaux, la violence et l'alcoolisme entre autres, qui minent encore ce peuple dépendant.

Néanmoins, le narrateur n’y parvient pas. Il ne maîtrisera jamais véritablement le groenlandais. Mais il voit, entre les lignes, les possibles significations symboliques de ce que cela signifie de ne pas maîtriser, surtout dans la perspective groenlandaise. La question de la langue est une question politique : «Le prétérit ordinaire n'existe pas. On dit : "Je le rencontre hier". " Grammaticalement, le passé est une forme du présent. Dans un sens figuratif, transposé au niveau de l'histoire politique, c'est un présent sans fin, la réalité d'un enfermement qui perdure. On ne sort pas de ce passé qui n'est rien sinon présent. Hier, tout comme il y a deux siècles et demi, c'est cela le passé : la condition de dépendance dans laquelle se trouvent emprisonnés les Groenlandais. Pourtant, d'autres formes du passé existent, par exemple « le plus-que-parfait transitif hypothétique négatif, etc. " $\mathrm{Si}$ je n'avais pas eu à le rencontrer" ". Mais ce n'est qu'une forme hypothétique négative. De nouveau, le passé reste le passé, puisqu'il est le présent, temps grammatical ici figurativement équivoque dans le sens où le passé ne lui échappera jamais.

2 Kim Leine, Kalak. Erindringsroman, $2^{e}$ éd., Copenhague, Gyldendal, 2015, p. 89-90. Toutes les traductions sont les miennes, sauf indication contraire. 
Les deux exemples de phrase - celui du passé qui est le présent ainsi que celui de la situation hypothétique négative d'un plus-que-parfait -, évoquent une rencontre. Une rencontre avec qui ? Probablement avec l'homme blanc tout de noir vêtu, l'homme noir luthérien colonisateur. Oui, l'homme groenlandais "le rencontre hier ». Il le rencontre aujourd'hui. Il le reverra. Si seulement les Groenlandais n'avaient " pas eu à le rencontrer ", le passé aurait pu être autre que le présent qu'il est. Mais ce n'est pas le cas.

Tout au long de mon article, j'étudierai donc les différentes perspectives que propose Kim Leine sur l'histoire coloniale qui lie le Groenland et le royaume de Danemark, en mettant l'accent sur Les prophètes du fjord de l'Éternité. La figure de l'hermaphrodite sera d'importance fondamentale, car elle englobe chez l'auteur tous les niveaux décisifs de la réflexion : forma duplex chez Ovide, cet équivalent mythologique de la contradictio in terminis logique incarne symboliquement dans l'œuvre de Leine toute la complexité de la question de l'identité et ce, tant au niveau individuel qu'au niveau culturel collectif. Impossible coprésence de deux éléments catégoriquement différents, l'hermaphrodite ne " peut " pas exister, ne " devrait " pas exister ${ }^{3}$. Pourtant, il est, et en tant qu'image, il porte depuis des millénaires une profonde complexité culturelle. Ainsi, chez Leine, il est lié au problème de l'" image de soi ", "selvbillede », et de l'identité : la vérité du "soi-même " et de l'identité - individuelle et culturelle - est à chercher dans la coprésence des formes doubles, voire antinomiques qui les constituent. Ce motif dans l'œuvre de l'auteur est de son côté associé à la question de la relation entre le bourreau et la victime. De nouveau, cette relation se développe à la fois sur le plan personnel et au niveau collectif, c'est-à-dire aux niveaux historique, culturel et social. À chaque victime son bourreau : il y a le "Kim Leine " victime d'un bourreau prédateur sexuel familial - à savoir l'auteur-narrateur du premier ouvrage, Kalak, qui expose entre autres les abus que lui faisait subir son père pendant l'adolescence - et il y a le peuple groenlandais, victime de la mainmise coloniale de son bourreau historique danois, comme le démontrent, sous forme historico-littéraire, les romans Kalak, Tunu, Les prophètes du ford de l'Éternité et Homme rouge/Homme noir.

3 Soulignons que ce propos est à entendre uniquement au niveau de la réflexion philosophique symbolique chez Kim Leine, niveau auquel l'hermaphrodite représente ce qui semble être une impossibilité logique. Dans une perspective plus générale, notons également que la relation entre les sexes dépasse largement le propos ici : logiquement, sinon historiquement, elle ne saurait bien évidemment être contraire. Voir à ces sujets par exemple Anne Fausto-Sterling, "The Five Sexes. Why Male and Female Are Not Enough ", The Sciences, mars-avril, 1993, p. 20-24 et "The Five Sexes, Revisited », The Sciences, juilletaoût 2000, p. 19-23. Voir Les cinq sexes. Pourquoi mâle et femelle ne sont pas suffisants, trad. d'A.-E. Boterf, Paris, Payot (Petite Bibliothèque Payot), 2013. Pour la citation d'Ovide, voir Métamorphoses, livre 4, v. 375-379, trad. et notes de A.-M. Boxus et J. Poucet, Bruxelles, Bibliotheca Classica Selecta, 2006, [http://bcs.fltr.ucl.ac.be/METAM/Met04/M04-274-415.htm]. 
Puis, parfois on devient victime de soi-même. Comment, dans ce cas, établir la culpabilité du bourreau ? La relation victime-bourreau constitue-t-elle une forme inquiétante d'hermaphrodite : un l'un-dans-l'autre aussi intime que contradictoire ? Toujours d'une précision crue, le travail littéraire de Leine s'interroge ainsi sur ces constellations complexes en n'oubliant jamais la considération de ce qu'on pourrait nommer la suprême réussite de la stratégie perverse du bourreau : parvenir à convaincre sa victime que cette dernière a en réalité désiré, et activement cherché, son propre destin. En outre, le motif de l'hermaphrodite - et donc de la relation victime-bourreau - est chez Leine également lié à la réflexion sur le temps, le temps individuel et historique : pour les victimes - "Kim Leine » et le peuple groenlandais -, le présent n'est peut-être que la blessure encore ouverte du passé, une relation étroite déterminée par la violence du lien intime même. Le passé est le présent et il constitue par conséquent - avec une autre figure - une prison d'une redoutable duplicité de laquelle on n'arrive apparemment pas à s'arracher.

\section{LES POIDS DE L'IDENTITÉ}

Un mercredi soir, fin octobre 2013, l'image de soi que porte Kim Leine - et qui le porte - est ébranlée. Sous les applaudissements du public, dont ses collègues nommés, l'auteur monte sur la scène de l'opéra d'Oslo afin de recevoir le prix littéraire du Conseil nordique pour Les prophètes du fjord de l'Éternité. "Je n'arrive pas à me voir en tant que quelqu'un de populaire ", dira-t-il un mois plus tard à la journaliste Mette-Line Thorup dans un entretien accordé au quotidien danois Information 4 . Il y a l'image que nous avons de nous-mêmes. Puis, il y a l'image, ou les images, que nous choisissons de donner à voir aux autres. Ensuite, il y a les images, multiples et variées, qu'ont de nous les autres. "Est-ce possible, en un instant, de changer complètement de position ? ", se demande l'auteur au cours du même entretien en réfléchissant sur les fondements historiques de l'identité :

L'idée d'une image de soi fixe et ferme est le joug de l'homme occidental. Les autres peuples n'en sont pas forcément porteurs. Je vois chez les Groenlandais qu'ils n'ont pas une image fixe d'eux-mêmes et cela les rend beaucoup plus dynamiques. Ils peuvent se transformer en très peu de temps. Tu peux rencontrer un Groenlandais qui te fait penser «Zut, celui-là n'en a plus pour très longtemps... ", puis qui disparaît pour ressurgir quelques mois plus tard, parfaitement cool et cadre dans une entreprise. Il s'est " morphé » en un tout autre état. Une telle capacité de transformation présuppose qu'on n'a pas d'image de soi trop fixe et solide. ${ }^{5}$

4 Mette-Line Thorup, "Han gør splittelsen synlig », Information, 5 décembre 2013, [https://www. information.dk/indland/2013/12/goer-splittelsen-synlig].

5 Ibid. 
Pour Leine, l'homme n'est jamais une seule et même chose. Mais qui est ce Groenlandais qu'on peut ainsi rencontrer, d'abord dans un état lamentable, faisant craindre le pire, puis parfaitement métamorphosé, seigneur dans son domaine ? Il n'est personne. Il est tout le monde. Ce n'est qu'un exemple. De cette manière, ce peut être n'importe quel Groenlandais. Sous le regard mi-imaginaire, mi-réel de Leine - un Kim Leine entre le « fait » et la « fiction » - un Groenlandais "victime " de sa situation, de son destin, de l'histoire coloniale, de sa famille, de son manque de chance... se transforme donc en un Groenlandais " maître » de sa situation, de son destin, sinon de l'histoire coloniale, de sa famille, du manque de chance qu'il a eu... «Il » peut toujours changer. « Tu » n'as pas cette aptitude, en tant qu'homme occidental. " Nous ", les Occidentaux, n'avons pas cette capacité. Nous restons nous-mêmes pour le pire et pour le meilleur.

Ce n'est pas seulement l'idée d'une « image de soi fixe et ferme » qui vient de "l'homme occidental » en constituant le « joug " de ce dernier. La conception de la distinction entre " eux " - l'homme sauvage, le sauvage noble, le peuple naturel ou primitif, à titre d'exemple - et " nous " dans le monde civilisé est également une invention occidentale. À travers les âges, le regard sur les " autres " change évidemment : des sauvages ou «brebis égarées » dont on s'étonne à la Renaissance après la découverte des Amériques jusqu'aux « primitifs » de l'anthropologie du $\mathrm{XX}^{\mathrm{e}}$ siècle, en passant par le "sauvage noble " célébré par les Lumières et les Naturvolk, les " peuples naturels " qui ont fait rêver les romantiques de chez "nous " - c'est-à-dire de chez nous en tant que Kulturvolk - autour de $1800 \ldots{ }^{6}$. Mais ce regard ne change que pour rester sensiblement le même : l'autre, ou l'Autre, est autre.

En un rien de temps, nous dit Leine, un Groenlandais peut transformer radicalement son destin, et ce, parce qu'il n'est pas comme "nous ». Mais si c'est effectivement le cas - et l'on n'a pas vraiment moyen de le savoir - cela est également, faut-il ajouter, son problème, étant donné que c'est encore «nous » - «nous », le Danemark et, à travers l'OTAN, les États-Unis - qui définissons largement ce que peuvent ou ne peuvent pas faire les Groenlandais. Ces propos de Leine - grand connaisseur s'il y en a des affaires groenlandaises passées et actuelles - montrent à quel point il est difficile pour un commentateur danois d'échapper au discours condescendant vis-à-vis de ce " territoire rattaché » du royaume de Danemark qui bénéficie depuis 2009 d'une " autonomie élargie ». Lauteur sait mieux que personne que le désir d'indépendance fait partie de l'identité " fixe et ferme " actuelle groenlandaise.

6 Voir à ce sujet, spécifiquement par rapport aux Groenlandais sous le regard danois, l'« Indledning " de l'éditeur dans Grønland - en refleksiv udfordring. Mission, kolonisation og udforskning, O. Høiris (dir.), Aarhus, Presses universitaires d'Aarhus, 2009, p. 9-39. 


\section{KalaK : UN VRAi Groenlandais EST UN SALE Groenlandais}

"Grønlanderstiv " est un adjectif danois attesté officiellement depuis 1997. On peut le traduire par " raide comme un Groenlandais " ${ }^{7}$. Si la certification officielle dans une langue d'un nouvel adjectif est significative - et tout porte à le croire - de la manière dont ses locuteurs regardent généralement la qualité qui y est exprimée, les Danois se doivent encore de réfléchir sur la nature des liens qu'ils entretiennent avec leurs concitoyens de l'ancienne colonie arctique.

"Kalak" est un substantif inuit qui dénote un "vrai Groenlandais ". Mais que signifie effectivement ce lexème, (un) vrai Groenlandais ? Cela dépend de la perspective. Selon le sens commun danois, un " vrai Groenlandais » est un "sale Groenlandais » : incapable de prendre sa vie en main, le Groenlandais, le vrai, est un cas social coûteux, un être immature et alcoolique qui a des relations sexuelles avec ses proches. Toutefois, le terme reste groenlandais. "Kalak» est un mot eskimo-aléoute, un mot inuit, et personne au Danemark ne connaît la langue groenlandaise. Les lecteurs de romans danois découvrent le terme en 2007, à la parution de Kalak. Roman de mémoires de Kim Leine. Au cours de celui-ci, le narrateur - "Kim ", le Danois - se découvre en tant que réplique d'un vrai Groenlandais : autrefois victime d'inceste, il devient lors de son séjour sur l'île, avec femme et enfants, un irresponsable toxicomane et alcoolique qui utilise son pouvoir, en tant qu'infirmier masculin danois, pour séduire un certain nombre de femmes groenlandaises, elles-mêmes parfois déjà victimes de violences, d'abus sexuels, d'alcoolisme. "Karina ", note le narrateur à propos de l'une d'entre elles, "vit avec sa mère, ses deux sœurs et ses deux fils dont elle ne mentionne jamais le père, ou les pères ». Un jour, la mère de Karina prépare un repas de phoque. En évidant l'animal, elle offre une part particulière à l'amant danois de sa fille.

Elle découpe un gros morceau du foie et me le tend sur la pointe du couteau. Je l'accepte et mange, me forçant à mâcher avant d'avaler. Ça a le goût de sang et de noisettes.

Karina fait une grimace. Tu manges du foie cru?

Pourquoi pas?

Tu as du sang autour de la bouche. Je ne t'embrasserai plus jamais.

Je me penche au-dessus de l'évier, rince mes doigts et ma bouche et m'essuie avec un torchon. Le foie cru fait chaud au ventre comme de l'eau-de-vie.

Kalak, dit Karina. Groenlandais de merde. ${ }^{?}$

7 Au sujet de l'attestation du terme, voir le dictionnaire de la langue moderne danoise publié par "Det Danske Sprog- og Litteraturselskab », [https://ordnet.dk/ddo/ordbog?query=gr\%C3\%B8nl\%C3 $\% A 6 n d e r s t i v]$, site consulté en novembre 2018. Dans le grand répertoire des préjugés intra-nordiques, "grønlanderstiv" semble en danois avoir pris la relève de l'équivalent "svenskerstiv ", " raide comme un Suédois".

8 Voir l'entrée dédiée à ce terme dans le dictionnaire officiel du Secrétariat linguistique du Groenland, Oqaasileriffik : [https://oqaasileriffik.gl/dict/?lex=8911].

9 Kim Leine, Kalak, op. cit., p. 100. Citation précédente, ibid., p. 99. 
Intimement dégoûtée par sa propre culture - culinaire, en l'occurrence - telle que celle-ci se présente d'ordinaire aux yeux des Danois, la maîtresse groenlandaise renverse par sa remarque finale la situation de manière singulière : en avalant tout cru le foie du phoque, le narrateur hurle avec les loups - sauvages ou domestiqués -, ce qui ne fait que renforcer l'écart qu'il espérait voir aboli. Parfois, l'ouverture bienveillante constitue le comble de l'arrogance et de la naïveté : en engloutissant le foie, le narrateur prétend implicitement que c'est à lui, et à lui seul, d'effacer la distance qui le sépare des Groenlandais, parce que c'est seulement lui qui maîtrise celle-ci. Par là, il les colonise de nouveau et les dévore. Dans le même temps, il ne fait qu'avaler tout cru sa propre définition de ce qu'être groenlandais veut dire. Puis, ce que signifie le terme kalak, vrai Groenlandais, reste toujours à définir. Mais celui qui se fait violence afin de se conformer aux autres n'est clairement qu'un "Groenlandais de merde ». Bien entendu, tout cela n'est à son tour qu'une interprétation danoise d'un roman danois.

Après leur rupture et un long séjour estival au Danemark avec sa famille, le narrateur rencontre à son retour Karina dans les rues de Nuuk. "Mon dieu, comme je te déteste, dit-elle». Et leur relation reprend.

Je suis content de revoir ses amis, les jeunes gars aux visages balafrés qui me saluent cérémonieusement et me demandent comment c'était au Danemark.

Affreux.

Ils rient d'un air entendu. Ils ont leurs propres souvenirs du Danemark.

Kalak, disent-ils.

C'est ainsi qu'ils me nomment. Le Groenlandais. C'est mi-moqueur, mirespectueux. Je porte le nom avec fierté. ${ }^{10}$

Les souvenirs du Danemark sont effroyables. Cependant, le rire apparemment complice des jeunes Groenlandais fait tacitement entendre l'irrémédiable clivage qui sépare les différentes interprétations de ce qu'une mauvaise expérience au Danemark pourrait bien signifier : au Danemark, un Groenlandais ne peut être qu'un Groenlandais, un kalak, qui fait ce que font les vrais Groenlandais en étant assis ivres morts sur un banc public dans la capitale du royaume. Mais le narrateur de Kalak ne fera jamais partie des vrais Groenlandais. Ainsi, ces jeunes dotés d'expériences et de visages balafrés qui semblent rire en connivence avec le narrateur reprennent et détournent en vérité le mot groenlandais "kalak» et ce, en le prenant sur eux, pour eux, par mépris du mépris. De toute façon, le terme "kalak», "vrai Groenlandais », ne veut en soi rien dire. 


\section{ROUSSEAU ET L'HOMME LIBRE ENCHAÎNÉ : LEINE FACE À L'HISTOIRE DE LA DOMINATION DU GROENLAND}

L'été 1787, Morten Falck - le pasteur personnage principal fictif du roman Les prophètes du fjord de l'Éternité - quitte le Danemark pour le Groenland. Originaire de Norvège, il a fait ses études de théologie à Copenhague avant d'embarquer ainsi pour la colonie atlantique. En ce sens, il suit la même trajectoire que le pasteur colonisateur originel, Hans Egede, né dans les îles norvégiennes de Lofoten et lui aussi formé à Copenhague, l'établissement de la première université norvégienne n'étant autorisé par la royauté danoise qu'en 1812. Kim Leine, de son côté, entamera ce périple à la fin des années 1970 quand il arrive, adolescent, à Copenhague en provenance de sa région natale du comté de Telemark. Après avoir obtenu son diplôme d'infirmier, il part pour le Groenland en 1989.

Au cours de son voyage, Morten Falck lit. Il est un lecteur assidu des philosophes et autres auteurs de son époque, la fin du XVIII siècle. Il veut vivre les Lumières parmi les indigènes, dans l'obscurité blanche du Groenland, où il est donc envoyé dans le but de poursuivre l'œuvre missionnaire protestante initiée par Egede lors de son arrivée sur l'île le 3 juillet 1721. Si « l'apôtre du Groenland », féroce luthérien qu'il était, souhaitait mettre tout son "zèle bienveillant » à l'œuvre " pour le bien du Salut des pauvres Groenlandais ignorants » - comme il l'indique dans sa Relation traitant de la culture de ces derniers ${ }^{11}$ - Morten Falck, lui, voit l'ignorance de la population locale sous un autre jour. Certes, les Groenlandais semblent encore largement ignorer l'existence du dieu chrétien. Philosophiquement plus intéressante est leur ignorance de l'ignorance même : véritables enfants des Lumières n'ayant aucune notion des Lumières, les Groenlandais sont à tous égards innocents. Morten Falck n'a pas encore mis les pieds sur le sol de la colonie. Néanmoins, il connaît ses habitants. En un certain sens, il les a rencontrés dans l'œuvre d'un auteur, à savoir Jean-Jacques Rousseau, qui n'en a jamais parlé. L'été 1787, à bord du brick Der Frühling, le pasteur se penche de nouveau sur son auteur de prédilection.

[S] on écrivain préféré reste Rousseau. "L’homme est né libre et partout il est dans les fers! " Ces deux phrases exercent toujours un grand effet sur lui, le constat que c'est un " et » qui les relie et non un « mais ». Il n'y a pas de contradiction entre les deux déclarations. L'homme est né libre. Et il est dans les fers. Morten frissonne chaque fois qu'il y pense.

11 Hans Egede, Omstandelig og udforlig relation om den grønlandske missions begyndelse og fortsattelse, Copenhague, J. C. Groth, 1738, "Fortale », s. p. Disponible sur le site de la Bibliothèque nationale de Norvège : [https://www.nb.no/nbsok/nb/029aac2395869c8648e16c7845a970f9?lang=no\#0]. Sur le travail et l'œuvre du missionnaire dano-norvégien, voir Mads Fægteborg, "Hans Egede », in Grønlanden refleksiv udfordring, op. cit., p. 39-75. 
Il se dit que Rousseau, avec sa conception de la nature et de l'homme, est en partie responsable de sa présence ici, sous le pont de ce petit navire, en route vers un poste de missionnaire au milieu des sauvages. Il lui tarde de rencontrer des gens dans leur état de nature, des hommes libres, vierges [frie mennesker, uspolerede]. Peut-être a-t-il envie de trouver un état de nature en lui-même. ${ }^{12}$

"L'homme est né libre, et partout il est dans les fers. ${ }^{13}$ Le célèbre incipit du Contrat social de Rousseau possède en effet une considérable force paradoxale et ce, à plusieurs égards : né libre, l'homme est enchaîné. Clairement, il y a une discordance entre la situation naturelle de l'homme - la liberté qui lui est propre et sa condition sociale et politique actuelle d'être enchaîné. Comme le signale Morten Falck, Rousseau lie les deux - la situation naturelle et la condition actuelle - par une conjonction marquant l'union, " et ", pas l'opposition : " mais ", " cependant ", etc. Un simple " mais » aurait été plus adapté au propos, semble-til. En choisissant « et ", Rousseau fait peut-être entrer « les fers » dans lesquels se trouve actuellement l'homme au cœur de sa liberté présumée originelle : comme " l'état de nature " rousseauiste tel quel, la liberté naturelle de l'homme est irrémédiablement perdue, compte tenu de l'être irréversiblement social de ce dernier, être dont la seule liberté possible dans un État de droit est celle conditionnée par "les fers", les contraintes nécessaires, du pacte social même ${ }^{14}$.

Est-ce bien ainsi que Morten Falck entend la pensée anthropologique et politique de Rousseau ? Pas tout à fait. Le pasteur des Lumières imaginé par Kim Leine semble plutôt enclin à séparer les versants politique et anthropologique de la réflexion rousseauiste : "L'homme est né libre et partout il est dans les fers!" ", cite-t-il avec enthousiasme et frissons. Les frissons sont dus au fait qu'il n'y a pas de " contradiction entre les deux déclarations ". Ainsi : "L'homme est né libre. Et il est dans les fers ». Selon Falck, il y a donc deux propos, ou "déclarations », chez Rousseau : le premier propos est anthropologique et constate la liberté (présumée) naturelle de l'homme, tandis que le second est politique et signale son être-enchaîné actuel. Puis, les deux ne sont pas en contradiction l'un avec l'autre. Jusque-là, à la limite, on peut suivre la logique du pasteur. Mais Rousseau ne fait pas "deux déclarations ". Il fait un seul propos, "L'homme est né libre, et

12 Kim Leine, Les prophètes du ford de l'Éternité, trad. d'Alain Gnaedig, Paris, Gallimard (Du monde entier), 2015, p. 88. Le danois original est cité d'après Kim Leine, Profeterne i Evighedsfjorden, Copenhague, Gyldendal, 2012, p. 83.

13 Jean-Jacques Rousseau, Du contrat social, B. Bernardi (éd.), Paris, GF Flammarion, 2012 [1 ${ }^{\text {re }}$ éd., 1762], p. 42.

14 "Tel se croit le maitre des autres, qui ne laisse pas d'être plus esclave qu'eux. Comment ce changement s'est-il fait ? Je l'ignore. Qu'est-ce qui peut le rendre légitime? Je crois pouvoir résoudre cette question" (ibid.), écrit Rousseau dans les lignes qui suivent le propos sur l'homme libre dans les fers. $\mathrm{Nul}$ ne sait et nul ne saura jamais comment s'est fait le changement qui ouvre la voie à l'état civil. Autant se demander comment l'homme a acquis la parole. Là n'est pas, ou n'est plus, la question. Il s'agit de « rendre légitime » l'état civil (voir les notes de l'éditeur ibid., p. 179-180). 
partout il est dans les fers ", qui témoigne de l'imbrication, de l'un-dans-l'autre, de l'anthropologique et du politique. C'est en pensant aux Groenlandais, dans sa perspective rousseauiste bien à lui, que Morten Falck oublie cela. En fait, il oublie les Groenlandais eux-mêmes, et ce, à l'instant précis où sa réflexion les atteint. Pour le coup, il y a contradiction : "Il lui tarde de rencontrer des gens dans leur état de nature, des hommes libres, vierges». Pouvait-on véritablement voir et toucher "des gens dans leur état de nature " sur les côtes groenlandaises à la fin du XVIII siècle ? Passons sur l'aspect anachronique de cette question - que l'on pose donc en théorie à un personnage fictif datant du début du XXI ${ }^{e}$ - vis-à-vis de la situation des Inuits vers 1787 : l'état de nature de l'homme est une fiction théorique rousseauiste, l'homme étant toujours déjà socialisé, même - ou peutêtre surtout - quand il est désigné « sauvage " ou "vierge " sous le regard extérieur qui fait de lui un Autre. Pour les mêmes raisons, passons également sur le fait que le personnage du pasteur n'hésite pas à qualifier les Groenlandais colonisés d'« hommes libres ». La vraie ironie implicite dont fait ici l'objet Morten Falck se trouve à un autre niveau : les Groenlandais sont des " hommes ", "mennesker ", et en tant que tels ils sont " libres ", "frie », se dit le pasteur. Mais de tels hommes n'existent pas, selon la réflexion de Falck lui-même, interprétant Rousseau : si les Groenlandais sont des hommes, ils ne sont pas libres, puisque l'homme est partout dans les fers.

L'interprétation contradictoire que propose Morten Falck de la pensée de Rousseau fait de cette dernière un vecteur de préjugés. En ce sens, le philosophe est effectivement " en partie responsable " de la présence du pasteur " ici, sous le pont de ce petit navire, en route vers un poste de missionnaire au milieu des sauvages ». Et qu'en est-il finalement de ces «sauvages », les Groenlandais « libres, vierges ", dans l'interprétation philosophiquement défaillante de Falck ? Ils deviennent prisonniers de leur liberté présupposée : étant « libres, vierges ", "frie, uspolerede ", les Groenlandais sont hors du temps, anhistoriques et ainsi parfaits dans le sens originel du terme. La liberté immémoriale que leur assigne et impose Morten Falck rend-elle également, à l'inverse, les Groenlandais irrémédiables ? Positivement, puis négativement, Falck ne fait que projeter la " liberté " qui lui fait défaut à lui-même - cette liberté dont il ne peut que rêver - sur ces autres, les Groenlandais, qui par là se font enchaîner : qu'ils soient meilleurs ou pires que nous, ils sont irréversiblement différents.

Il est possible de partir en expédition dans l'unique but de ne pas se déplacer réellement. Cela porte le nom de voyage touristique, pratique peut-être plus ancienne qu'on ne le croie. Il est également possible de partir de chez soi afin d'imposer son monde à l'ailleurs qu'on découvre, puis l'exploiter. Ou bien on voyage dans l'espoir de mieux se découvrir en se déplaçant, c'est-à-dire dans 
l'espoir que le point de départ puisse avoir lieu véritablement ailleurs. Finalement, il y a le rêve de la pure découverte. En tant que parfait fantasme, celui-ci est nul et non avenu ${ }^{15}$.

\section{L'HERMAPHRODITE : LE BOURREAU, LA VICTIME, L'EXPÉRIENCE DU TEMPS}

Qu'ont cherché à faire les Danois, au Nord, vis-à-vis des Groenlandais ? À civiliser et à dominer, à libérer et à mettre dans les fers. Longtemps après sa lecture de Rousseau, Morten Falck pousse du haut d'un précipice une femme groenlandaise, entraînant sa mort. Nous sommes tout au début des Prophètes du fjord de l'Éternité et c'est déjà la fin de quelque chose, de quelqu'un. En réalité, tout récit commence par la fin de quelque chose : le prologue du roman de Leine, intitulé "La chute (14 août 1793) »- la veille de l'Assomption, six ans après l'arrivée du pasteur - narre donc la mort d'une femme, surnommée "la veuve ", qui se fait suicider par l'intervention d'un homme anonyme, un " il " qui s'avère plus tard être Morten Falck, la poussant dans l'abîme. Voici l'incipit : «La veuve est venue d'elle-même, nul ne l'y a forcée ${ }^{16}$. Puis, tout en haut de l'à-pic, elle se prépare pratiquement, moralement et religieusement - chrétienne, elle se fera inscrire post mortem sur la liste des baptisés par celui qui va la faire chuter - à ce qui va ou doit arriver. Elle s'assoit et elle attend.

Elle sent qu'il s'est arrêté à deux pas derrière elle et s'imagine qu'il la contemple comme la première fois qu' ils étaient ensemble. Il se demande où il va la frapper et avec quelle force, car elle sait qu'il veut la tuer, mais il ne veut pas lui faire du mal. C'est une consolation de le savoir si près au moment où sa vie est sur le point de prendre fin, cela la rassure et la détend, elle baisse le menton contre sa poitrine et inspire profondément. Fils de Dieu, nous te prions, écoute-nous! ${ }^{17}$

L'homme qui va tuer ne souhaite aucun mal à celle qu'il va faire disparaître. En fait, il s'approche d'elle " presque pudiquement, avec la gêne d'un jeune soupirant $»^{18}$. En effet, la veuve est depuis un certain temps sa maîtresse. Parallèlement, cette dernière trouve apaisant de le savoir "si près " au moment où elle va mourir, c'est-à-dire au moment où elle va mourir parce quìl est « si près " d'elle. Apparemment, il n'est pas un bourreau et elle n'est pas une victime. La veuve semble être son propre bourreau et Falck sa victime. En ce sens, la " chute » du titre du prologue est celle - intérieure, éthique - subie par le pasteur. Certes, la veuve tombe et meurt. Mais ce n'est pas une chute, puisqu'elle se laisse mourir en se faisant pousser.

15 Précisons tout de même qu'il est ici question de voyage, d'expédition... de connaissance et/ou de conquête de l'ailleurs... pas d'émigration forcée et d'exil.

16 Kim Leine, Les prophètes du fjord de l'Eternité, op. cit., p. 11.

17 Ibid., p. 12.

18 Ibid. 
Est-il question d'un meurtre doux ou d'un assassinat libérateur, si tant est que de tels actes existent ? La veuve est tiraillée, apprend-on au fur et à mesure, entre deux univers : d'un côté, la société traditionnelle des Inuits la rejette à cause de son statut - une veuve dans l'incapacité de subvenir à ses propres besoins était considérée comme un fardeau qui devait volontairement disparaître dans les montagnes, destin impensable pour une personne baptisée - et la société des Danois colonisateurs qui ne l'acceptera jamais véritablement, chrétienne ou pas ${ }^{19}$. Ainsi, elle finit par se faire supprimer par ce pasteur qui « ne veut pas lui faire du mal ». Nul ne pousse directement la veuve à se faire pousser. C'est juste ce qu'elle doit faire, libre d'être enchaînée comme elle est.

Encore étudiant, Morten Falck rencontre un personnage particulier dans un quartier mal famé de Copenhague. Ce dernier est un garçon qui est une fille qui est un garçon : un garçon qui est ce qu'il n'est pas. De quoi ce garçon vit-il ? Il « se déplace normalement avec une troupe de saltimbanques. Son travail, c'est de dire la bonne aventure ». Connaissant le passé de chacun, il sait prédire l'avenir. " Partout. N'importe où ", est là où il préfère être. En plus, c'est un voleur, faisant les poches des gens dans leurs moments d'inattention ${ }^{20}$. Falck finira par coucher avec lui, ou elle, payant la somme de dix marks. Mais d'abord il essaie de comprendre ce que représente cet être qui est nommé, selon les perspectives, le " garçon » ou la « fille » :

Morten le regarde fixement. Il voit la fille incrustée dans ses traits. Mais c'est le garçon qui parle.

"Mon bienfaiteur. Vous avez un cœur noble et distingué. Je le vois bien.

Vous n'avez pas à avoir honte.

- Je n'ai pas honte. Je me demande simplement ce que tu es.

- Venez avec moi jusqu'aux remparts, dit le garçon, et je vous montrerai. Ça coûtera dix marks. Quand vous en aurez fini avec moi, vous retrouverez la paix de l'âme. Vous redeviendrez un bon citoyen et vous recommencerez à faire la cour à vos filles, comme c'est votre devoir. " ${ }^{21}$

Ce garçon-fille ne devrait pas être. Il constitue, dans la considération de Morten Falck, une "inadmissibilité biologique "22. Il est contraire à lui-même. Mais il n’y a aucune contradiction. Il existe. Et il existe en tant que celui qui n'est pas une seule et même chose. En un autre sens, cela est exactement ce qu'il est : uniquement

19 En fait, avant d'accéder au précipice, la veuve est déjà victime et bourreau, ayant tué son propre père, un missionnaire danois violeur incestueux, qui est également le père de sa fille décédée, fille qu’elle espère retrouver après la mort (ibid., p. 432). Seule à connaître le secret de Falck - un soir, ivre et cherchant de l'alcool, il a mis feu à la maison du négociant de la colonie, causant la mort d'une personne -, elle l'entraîne avec elle en haut de l'abîme pour faire de lui son assassin.

20 Kim Leine, Les prophètes du fjord de l'Éternité, p. 45 et 57 . Sur l'hermaphrodite en tant que voleur, ibid., p. 62-63.

21 Ibid., p. 49-50.

22 Ibid., p. 49. 
unifié à travers son désaccord intime, son identité consiste à être différent de luimême. En effet, l'existence et l'histoire ne sont pas simples. En couchant avec l'hermaphrodite, Morten Falck pense inévitablement à Rousseau et ses deux propositions qui ne sont pas en contradiction :

Le garçon le conduit à un appentis. Il frappe un signal sur le mur. Un verrou est tiré, ils entrent à l'intérieur. Dans la petite pièce, une femme est en train de tricoter à la faible lueur d'une bougie. Elle ne lève pas les yeux. Ils passent dans l'autre pièce qui compte un grabat et un poêle. Rien d'autre.

"Vous voulez de la lumière? demande le garçon.

- Oui. Beaucoup de lumière. "

Le sang bat dans sa tête, il a la bouche sèche. Rien ne doit lui être inconnu, songe-t-il. "L'homme est né libre et partout il est dans les fers! " ${ }^{23}$

Homme éclairé, Morten Falck veut tout connaître et il lui faut par conséquent toujours plus de lumière. C'est un rite de passage : le garçon qui n'est pas un garçon, mais une fille qui n'est pas une fille semble incarner pour le futur pasteur l'être double de l'homme libre dans les fers. L'hermaphrodite est le mystère même, doté d'un pouls, d'un pénis et de seins de femme. Comment pourrait-on prétendre aux Lumières, à tout connaître, sans devenir intime avec lui ? En un sens, l'hermaphrodite est l'homme libre, l'homme qui dépasse l'homme. Cela dit, l'hermaphrodite est en même temps l'homme confiné à sa dualité, l'homme condamné à être toujours ce qu'il n'est pas. Mais qui est donc la femme qui tricote paisiblement dans l'antichambre de la révélation du mystère, en ne disant rien, sans regarder personne, et qui semble avoir déverrouillé la porte sans s'être déplacée ? Dans la mythologie et la littérature occidentales il existe un grand nombre de femmes qui tricotent ou manient dextrement les fils de la vie : des Parques, Moires ou - dans la mythologie nordique - Nornes qui déroulent le fil du destin jusqu'à $\mathrm{M}^{\text {me }}$ Defarge dans A Tale of Two Cities (1859) de Dickens qui tisse les noms de ses ennemis dans l'espoir de les livrer à un funeste sort, en passant par Pénélope qui pendant vingt ans défait la nuit ce qu'elle a fait le jour de la grande toile d'attente du retour de son mari, Ulysse, les exemples abondent. C'est une question de temps. Chez Kim Leine, dans Les prophètes du ford de l'Eternité, la femme qui tricote est la gardienne de l'hermaphrodite. Ainsi s'ouvre une nouvelle perspective pour la considération du rôle que joue dans le roman le propos de Rousseau, considération qui n'est pas nécessairement en accord avec l'interprétation qu'en fait Morten Falck. C'est la suivante : l'homme est né libre et dès le premier instant il est dans les fers de son passé.

Face à l'hermaphrodite, Falck est confronté à un seuil dans sa propre vie, voire dans le temps même : vis-à-vis de lui, il y aura à plusieurs égards un avant et un après. L'hermaphrodite représente effectivement une certaine conception du

23 Ibid., p. 50. 
temps : cet homme-femme est plus qu'un être intermédiaire, il est un l'un-dansl'autre, et ainsi il signifie la persistance du passé dans le présent, l'avenir du présent, ainsi que l'avenir du passé. C'est cela qu'il dévoile - en tant que représentant symbolique - dans ses conversations avec Morten Falck : il sait dire le temps - le passé, le présent, le futur - puisqu'il est le temps, ce temps qui est un voleur vagabond, subtilisant seconde après seconde la vie de chacun tout en étant lui-même partout et nulle part.

À l'époque moderne, dit-on souvent - peut-être trop souvent - le temps s'est accéléré. Ce qui nous fait manquer de temps ${ }^{24}$. Avec le développement des nouveaux moyens de transport et de communication, cette accélération va de plus en plus vite : après le carrosse, le train, après le train, la voiture, après la voiture, l'avion... Aussi, le téléphone multifonctions - qui donne accès à toute information presque en temps réel - a pris la place du téléphone traditionnel, qui avait lui-même pris la place du télégraphe, etc., comme le courrier électronique a pratiquement fait disparaître la correspondance traditionnelle par voie postale. À propos des lettres d'une autre époque, nous allons lire quelques passages des Prophètes du fjord de l'Eternité. Mais avant de faire cela, accompagnons Morten Falck au terme de son voyage initial au Groenland - fin qui coïncide exactement avec celle du deuxième chapitre de la première partie du roman, chapitre qui raconte le périple maritime du pasteur en route pour l'île. Morten Falck s'est enfermé dans sa cabine :

Il est allongé sur le dos sur sa couchette. Il a joint les mains. Il regarde fixement le plafond. La cabine et le bateau sont encore terre danoise, il court des fils invisibles d'ici au Danemark et, au fond, jusqu'à la maison de son enfance. Il n'est pas encore parti. Pas tout à fait. Il y a encore une chaîne, des fers. Mais dès qu'il descendra à terre, il sera libre. ${ }^{25}$

La terre de la liberté, c'est le Groenland, peu importe ce que pourraient en penser les Groenlandais. À travers ces réflexions, Morten Falck semble se situer dans les limbes de son espace-temps à lui, de son histoire à lui : il est arrivé ailleurs, tout en restant à l'endroit d'où il vient. C'est uniquement au moment où il mettra pied à terre qu'il sera parti. Le passé persiste. Mais en quoi consiste l'avenir de ce présent, de ce moment sur le bateau?

Un saut de page, et six ans se sont écoulés. À la fin du deuxième chapitre de la première partie, on était le 16 août 1787 . Au début du troisième chapitre, on est arrivé à la date du 15 août 1793. C'est donc le lendemain de la mort de la veuve,

24 Voir par exemple Hartmut Rosa, L'accélération. Une critique sociale du temps, trad. de Didier Renault, Paris, La Découverte (Sciences humaines et sociales), 2010 (Beschleunigung. Die Veränderung der Zeitstrukturen in der Moderne, Francfort-sur-le-Main, Suhrkamp, 2005).

25 Kim Leine, Les prophètes du fjord de l'Éternité, op. cit., p. 116. 
décrite dans le prologue du roman. En l'espace d'une seconde - pour le lecteur l'avenir radieux de Morten Falck, l'avenir de sa liberté, s'est transformé en un trou temporel, qui ne sera rempli que plus tard, graduellement, et ce, par les malheurs qui constituent son futur réel, futur encore inconnu pour le lecteur. Que fait le pasteur missionnaire, au début de ce troisième chapitre ? Il guette le bateau qui doit le ramener au Danemark. Tous les espoirs liés à son séjour groenlandais se sont effondrés : alcoolique, malade, il ne rêve que de retrouver son point de départ scandinave.

Mais il fallait parler de lettres, de la correspondance écrite d'autrefois. Aux premières pages de ce troisième chapitre, plusieurs lettres - fictives, faisant partie de l'univers imaginaire du récit - sont citées. Et dans le présent de Morten Falck - ce 15 août 1793 -, ces lettres constituent des témoignages écrits sur son propre passé, et sur celui de ses proches. Ce qui revient à dire qu'il faudrait en réalité écrire " passé » au pluriel : il y en a toujours plusieurs.

La première lettre est une courte note, envoyée par le père de Morten Falck deux ans auparavant, qui annonce le décès de la mère du pasteur. La seconde lettre est celle que Falck lui-même a adressée à sa direction à Copenhague, demandant un "remplaçant " pour sa charge. La troisième lettre est un envoi non daté, qui relate la vie malheureuse de sa sœur dans la petite ville de Nakskov, au Danemark :

\section{Mon cher frère Morten,}

Ici, à Nakskov, l'air est épais, brumeux et malsain depuis le jour de Noël, avec à peine un rayon de soleil chaque jour, ce qui ne correspond guère à notre climat à Lier, où l'air était presque toujours clair, avec du soleil et un temps agréable. En ce qui concerne ma personne, nous allons bien, comme tu as pu le constater de tes propres yeux, nous menons une existence modeste, oui, plus que cela, mais je regrette et je regretterai peut-être toujours Lier, notre bon père et notre mère bienaimée, et le cimetière avec les belles tombes de nos frères et sours. J'ai éprouvé plus de joie dans les conditions de vie simples d'autrefois qu'aujourd'hui dans cette ville fanfaronne et imbue d'elle-même où je ne trouve guère d'agrément, et où, après sept ans de mariage, je ne me sens toujours pas chez moi. Que Dieu me pardonne mon langage!

Mon cher Morten, je me demande parfois si nous reverrons jamais notre père et la maison de notre enfance. Et te reverrai-je, mon frère adoré ? ${ }^{26}$

Peut-être la voix que l'on entend dans cette lettre est-elle une voix d'outretombe. La sœur parle de son présent gris et brumeux - un présent qui est actuellement loin dans le passé, étant donné que le facteur, le bateau, ne vient qu'une fois par an -, et elle compare ce présent à un " autrefois " bien plus heureux et lumineux - mais également marqué par la mort, déjà - qu’elle $a$ partagé, et qu’elle

26 Ibid., p. 121. Pour les deux lettres précédentes, ibid., p. 118 et 119. 
partage encore avec son frère, Morten Falck. Il n'y a pas un passé, il y a toujours plusieurs passés : chaque moment est multiple, selon les perceptions individuelles de celles et de ceux qui en font l'expérience, ou qui l'ont vécu. C'est cela, le passé historique : chaque instant est infini. Morten Falck ajoute le commentaire suivant à la lettre de sa sœur :

Un bateau peut être chargé d'avenir, tout comme il peut être chargé par le passé, par du courrier qui déterminera une action ou une autre, par des messages sur ce qui s'est passé l'année dernière au pays, réduits à une lecture fébrile d'une demi-heure à peine. ${ }^{27}$

Il y a les temps, au pluriel, de n'importe quel moment. Et pour Morten Falck il y a également les temps décalés de la lecture dans l'ailleurs : dans sa situation, avec chaque arrivée du bateau, le passé a un avenir, au sens propre du terme. Puisque pour le pasteur le passé est à venir au sens littéral de l'expression, c'est-à-dire pas encore arrivé - chez lui, au Groenland. Et quand le passé arrive, il est toujours écrit au présent.

\section{LE PRÉSENT ABSOLU}

Comment fait-on récit, c'est-à-dire comment fait-on pour rendre présent le passé ? "Il s'efforce de supprimer la distance entre l'écrit et ce qui est décrit par ce qu'il écrit ", note le narrateur au sujet de l'écriture du journaliste et auteur Kaj Gottlieb, l'un des frères jumeaux personnages principaux du roman L'abîme: "Écrire dans le présent absolu. Il y réussit presque ${ }^{28}$. Comment atteindre ce "présent absolu » ? Écrire temporellement à même ce qui est décrit par l'écriture. Regardons à l'œuvre le narrateur des Prophètes du fjord de l'Éternité, suivant au plus près les faits, les gestes et les pensées de Morten Falck, pasteur et artiste graphique à ses heures.

Quand il rentre du déjeuner, il fait nuit depuis longtemps. La veuve est couchée dans son alcôve, il distingue le contour de sa hanche arrondie sous la couverture. Il soupire et s'assied avec le Christiania-Kureren, allume la lampe. La veuve s'active avec les casseroles dans la cuisine. Va-t'en! [Gå nu vak, «Vat'en à présent "] songe-t-il, fatigué.

Il ouvre le tiroir et sort les dessins d'elle qu'il a faits de mémoire. La plupart sont des études de son visage, tentatives de la saisir avec le minimum de traits. Il y a également quelques nus détaillés, dessinés avec lenteur et émotion. En réalité, il ne l'a jamais vue nue. Ce qu'il a dessiné, c'est ce qu'il désirait voir, une sauvage qu'il a capturée et tenté d'apprivoiser. Les dessins ne sont pas

27 Ibid., p. 121.

28 Kim Leine, L'abîme, trad. d'Alain Gnaedig, Paris, Gallimard (Du monde entier), 2018, p. 397. Je corrige la traduction, le traducteur ayant mis " conserver " pour le danois " ophave ", "supprimer ", ce qui crée de toute évidence un contresens flagrant. Voir Kim Leine, Afgrunden, Copenhague, Gyldendal, 2015 , p. 379. 
particulièrement bons. Ses mains commencent à trembler, son trait n'est plus aussi sûr qu'autrefois. Il déchire les feuilles et en jette les morceaux dans le seau de charbon où elles s'enflamment et se ratatinent. Voilà, elle a disparu $[\mathrm{Nu}$ er hun vak, "Maintenant, elle n'est pas là »]. Il ne reste que la bouteille et le Christiania-Kureren [Nu er der flasken og Christiania-Kureren, " Maintenant il y a la bouteille et Christiania-Kureren "]. ${ }^{29}$

Pendant longtemps, il a essayé de la saisir. À présent, il souhaite la faire disparaître. C'est le passé, mais c'est écrit au présent grammatical. Le roman de Kim Leine n'est pas un récit minimaliste, loin de là. Mais sa manière de rendre présent le ou les temps des personnages a certains aspects en commun avec la technique graphique que recherche Morten Falck : il faut saisir le temps avec « le minimum de traits ". La veuve est là, elle est présente. Le pasteur aimerait bien qu'elle s'en aille maintenant. Puisqu'elle ne s'en va pas, mais s'obstine à rester - quoique toujours à distance, ailleurs même à l'intérieur -, Falck se tourne vers les dessins, enfouis dans un tiroir, qu'il a faits d'elle dans le passé. Ces dessins, il les a faits « de mémoire ». Il est donc question d'un passé du passé. Dans ce passé, Morten Falck a essayé d'étudier son "visage ", et ainsi de la saisir, elle, avec " un minimum de traits ». Un visage n'étant que ses propres traits, le défi a été de taille. Puis, il y a des nus détaillés, faits avec «lenteur et émotion », comme pour arrêter en douceur cette femme fugace et aérienne. Mais, finalement, Falck n’a jamais dessiné que ce qu'il désirait voir : une femme "sauvage ", "capturée " et domestiquée. Mais elle lui a échappé, elle lui échappe, elle lui échappera toujours. Les nus de cette femme qu'il n'a jamais vue nue sont entre le fait et la fiction : un rêve ourdi à partir de bribes de la réalité. Regardons ce qui arrive quand Morten Falck jette ses dessins sur le feu : "Voilà, elle a disparu ", " $N u$ er hun vak». Le " vak» danois est un adverbe qui exprime le fait que quelqu'un ou quelque chose n'est pas dans un lieu (quelconque) : "Maintenant, elle n'est pas là». Maintenant, il ne reste pour Falck que la bouteille, c'est-à-dire l'oubli, et Christiania-Kureren, journal relatant les événements de l'ici-maintenant de la capitale norvégienne que le pasteur reçoit toujours avec un an de retard.

En réalité, Falck s'égare un peu. Il se trompe, peut-être à cause de la présence de la bouteille. Puisque la veuve est encore là. Ce que Morten Falck fait disparaître d'elle est sa représentation. Elle, la veuve, reste, en même temps à portée de main et inaccessible. Elle est comme l'instant du récit : la présence de l'absence, parce qu'elle est le présent, rempli de passé. Quand on la touche, elle est déjà partie, passant par-dessus le précipice dans un suicide assisté qui n'en est pas un. La veuve groenlandaise est le moment présent qui ne vient jamais, mais qui est toujours, d'un instant à l'autre. Ainsi, elle ne cesse de disparaitre, en nous maintenant près

29 Kim Leine, Les prophètes du ford de l'Éternité, op. cit., p. 344 et Kim Leine, Profeterne $i$ Evighedsforden, op. cit., p. 324. 
d'elle. Il n'est donc pas surprenant, après tout, que les nus dessinés par Morten Falck ne soient pas réussis : "En réalité, il ne l'a jamais vue nue ». Le présent est ce que personne n'a vu $n u$ : le perpétuel maintenant, pas là, $n u$, vak, qui restera toujours veuf puisqu'il nous fuit et nous enterre juste en étant là, impossible à apprivoiser ${ }^{30}$. De toute manière, dans le temps, personne n'est chez soi. Pourtant, le temps est le foyer de chacun.

\section{LE NO MAN'S LAND DU GROENLAND, UN CHEZ-SOI}

Bien avant d'avoir ainsi dessiné la veuve, puis brûlé sa représentation, Morten Falck quitte l'appentis où il a couché avec le garçon-fille, appentis situé aux remparts de Copenhague, c'est-à-dire sur un terrain vague : « ... il remet de l'ordre dans ses vêtements, salue poliment la femme dans le couloir et rentre chez lui par le no man's land [ingenmandslandet] $"{ }^{31}$. Oui, Falck salue avec politesse la Norne et rentre chez lui par le no man's land, "ingenmandslandet " - " la terre d'aucun homme "-, qui est le territoire du temps et de l'histoire hermaphrodites. Puis, il part pour le Groenland où il mourra à l'âge de presque 51 ans. En groenlandais, le Groenland est nommé Kalaallit Nunaat : la Terre des Hommes, c'est-à-dire la Terre des Groenlandais. Tout comme kalak, un "vrai Groenlandais ", le terme Kalaallit Nunaat ne veut rien dire en soi : une terre qui appartient aux hommes est forcément un no man's land-au moins jusqu'à l'arrivée, le jeudi 3 juillet 1721, de ceux qui créent les Autres, sauvagement libres, disponibles pour être colonisés. Les Danois ne quitteront pas le Groenland de sitôt, ayant toujours besoin de ces Autres afin de circonscrire leur propre liberté, illusoire ou pas, ta liberté à toi étant à chercher dans ce que tu n'es pas. Le rêve de l'ailleurs, de la conquête de l'ailleurs, est aussi le rêve de la maîtrise du temps et de l'histoire. "Tu n'as qu'à prendre la voie du Nord, mon ami, tôt ou tard tu trouveras ton chez-toi ", estil dit dans la dernière phrase du roman Homme rouge/Homme noir ${ }^{32}$. Le propos est équivoque, doté à la fois de pathos et d'ironie. Il est également irréfutable, puisque tautologique : oui, celui qui se met en route vers le Nord trouvera tôt ou tard son chez-soi, ne serait-ce qu'en retournant à son point de départ. Ainsi, même les Groenlandais trouveront un jour leur chez-eux en prenant le chemin du Nord, étant donné que depuis presque trois siècles ce foyer est situé dans le Sud. L'homme est né libre, et partout il est dans les fers.

30 J'écris cela au masculin surtout parce qu'il n'y a pas de genre masculin, ni féminin en danois : il n'y a que le genre neutre et le genre commun. Le mot "veuve ", " enke ", appartient au genre commun.

31 Ibid., p. 51. Kim Leine, Profeterne i Evighedsforden, op. cit., p. 49.

32 Kim Leine, Rød mand/sort mand, Copenhague, Gyldendal, 2018, p. 512. 
